\title{
Study on the High Accuracy and Fast Acquisition of Satellite Signals Based on the Blind Source Separation Technique
}

\author{
Bo Li $\left(\mathbb{D},{ }^{1}\right.$ Kan Xie $\mathbb{C D}^{1,2}$ Yulei Bai, ${ }^{1,3}$ Zongze Wu, ${ }^{1,4}$ and Shengli Xie ${ }^{1,5}$ \\ ${ }^{1}$ School of Automation, Guangdong University of Technology, Guangzhou, China \\ ${ }^{2}$ Key Laboratory of Intelligent Information Processing and System Integration of IoT (GDUT), Ministry of Education, \\ Guangzhou, China \\ ${ }^{3} 111$ Center for Intelligent Batch Manufacturing Based on IoT Technology (GDUT), Guangzhou, China \\ ${ }^{4}$ Guangdong Key Laboratory of IoT Information Technology (GDUT), Guangzhou, China \\ ${ }^{5}$ Guangdong-HongKong-Macao Joint Laboratory for Smart Discrete Manufacturing (GDUT), Guangzhou, China
}

Correspondence should be addressed to Kan Xie; kxie@gdut.edu.cn

Received 14 October 2021; Revised 22 November 2021; Accepted 27 December 2021; Published 17 January 2022

Academic Editor: Punit Gupta

Copyright $\odot 2022 \mathrm{Bo}$ Li et al. This is an open access article distributed under the Creative Commons Attribution License, which permits unrestricted use, distribution, and reproduction in any medium, provided the original work is properly cited.

To address the problems of slow acquisition speed and low accuracy faced by existing grid search-based satellite acquisition methods in complex scenarios, this study proposes a high accuracy and fast satellite signal acquisition method based on blind source separation. The proposed method first adopts wavelet threshold denoising to reduce the noise in the overlapped satellite signal received by the receiver so as to improve the signal-to-noise ratio of the satellite signal. On this basis, a subspace estimation method is introduced to construct a blind acquisition algorithm for satellite signals, which achieves high accuracy and fast solution for the Doppler frequency shift and code phase shift of satellite carrier signals and is able to recover the unobservable individual source signals. The effectiveness of the proposed method is verified through experiments and compared against the traditional grid search type acquisition method, which confirmed that the method is suitable for the acquisition requirements of weak signals and has a good engineering application value.

\section{Introduction}

Satellite signal acquisition is an important part of the receiver baseband signal processing, which is a prerequisite for tracking and positioning. The performance of the acquisition algorithm directly affects performance metrics such as the receiver acquisition speed and accuracy $[1,2]$. For a particular GPS satellite signal, signal acquisition is the estimation of the carrier frequency and code phase of the satellite signal [3-5]. Previous research has proposed to use a parallel search algorithm for Doppler frequency and code phase on a two-dimensional grid [6]; however, as finer grid divisions are required to improve acquisition accuracy, while coarser grid divisions are required to improve acquisition speed, this method cannot simultaneously optimize both the acquisition speed and accuracy. Cui et al. [7] also proposed a matched filter coarse acquisition and linear frequency modulation $Z$-transform fine acquisition method that improves the acquisition speed while maintaining a certain degree of satellite signal acquisition accuracy; however, the method has a relatively high complexity and has poor acquisition performance in complex scenarios where the signal-to-noise ratio is low. Although many studies on satellite signal acquisition techniques have been performed, no method to date can achieve both fast and accurate satellite signal acquisition in complex scenarios.

Based on the existing research, this study proposes a high accuracy and fast acquisition method for satellite signals based on blind source separation $[8,9]$. The proposed method first introduces the wavelet denoising method to reduce the background noise of the satellite signal. Subsequently, the blind source separation model of the satellite signal is constructed by frequency multiplication sampling, and the subspace estimation theory is introduced to resolve 
and compute the Doppler frequency shift and code phase shift. Finally, the acquisition performance of the proposed method is evaluated based on actual GPS satellite signals and compared against that of the traditional parallel search method.

\section{Methodology}

2.1. Blind Acquisition Model of Satellite Signals. The satellite signal received by the receiver contains 3 aspects: the carrier wave, the pseudo-random code, and the data code. Each satellite corresponds to a unique pseudo-random code, i.e., the $\mathrm{C} / \mathrm{A}$ code. The $\mathrm{C} / \mathrm{A}$ code and the data code are modulated into a combination code, and the low code rate combination code is secondarily modulated using spread spectrum communication techniques to form the final satellite signal as follows:

$$
r(t)=\sum_{i=1}^{N} A_{i} \cdot C_{i}(t) \cdot D_{i}(t) \cdot \cos \left[2 \pi \cdot\left(f_{I F}+f_{i}\right) \cdot t+\theta_{i}\right]+n(t) .
$$

In the above equation, $N$ is the total number of satellites received by the receiver, time $t$ denotes the continuity of the satellite signal, $A_{i} i$ expressed as the amplitude of the $i^{\text {th }}$ satellite's carrier signal, $C_{i}(t)$ corresponds to the C/A code of the $i^{\text {th }}$ satellite, $f_{I F}$ represent the carrier frequency, $f_{i}$ and $\theta_{i}$ are, respectively, the Doppler frequency shift and initial phase of the $i^{\text {th }}$ satellite's carrier signal, the solution involving these two parameters corresponds to the acquisition of the satellite, $D_{i}(t)$ is the $i^{\text {th }}$ satellite's navigation data code, and $n(t)$ is the background noise.
The solution of Doppler frequency shift and code phase shift in equation (1) can be reduced to a single-channel blind source separation problem, i.e., separating each satellite carrier signal from the mixed carrier signal received by the receiver and then solving for the Doppler frequency shift and code phase. However, without any a priori knowledge, the single-channel blind source separation is a pathological computational problem. As such, this study converts the satellite signal into an aliased sinusoidal sampled signal with multichannel output through frequency multiplication sampling. The sampling points of each channel are different during the sampling process, ensuring that there is variability in the information observed by each channel, thus adding new observational information. Because C/A codes and navigation data codes are digitally coded signals, when sampling is sufficiently dense, the values of $\mathrm{C} / \mathrm{A}$ codes and navigation data codes are constant across different channels for a given point in time. Based on this train of thought, considering only a particular sampling point and assuming an evenly spaced distribution of sampling points between channels for a given point in time, the satellite signal reception model after channel serialization can obtained as

$r(\rho)=\sum_{i=1}^{N} A_{i} \cdot P_{i} \cdot \cos \left[2 \pi \cdot F_{i} \cdot \frac{t_{M}}{M-1} \cdot(\rho-1)+\theta_{i}\right]+n(\rho)$,

where $P_{i}=C_{i}(t) D_{i}(t)$ and $F_{i}=f_{I F+} f_{i}, j=1,2, \ldots, M$ are the sampling channels, and $t_{M}$ is the sampling time corresponding to the $M^{\text {th }}$ sampling channel. Rewriting equation (2) in matrix form by means of Euler's formula,

$$
\begin{aligned}
& \mathbf{r}=\mathbf{A} \cdot \mathbf{x}+\mathbf{n}, \\
& \mathbf{A}=\left[\begin{array}{cccc}
1 & 1 & 1 \\
\exp \left(j \cdot 2 \pi F_{1} \cdot \frac{t_{M}}{M-1}\right) & \exp \left(-j \cdot 2 \pi F_{1} \cdot \frac{t_{M}}{M-1}\right) & \cdots & \exp \left(-j \cdot 2 \pi F_{N} \cdot \frac{t_{M}}{M-1}\right) \\
\exp \left(j \cdot 2 \pi F_{1} \cdot \frac{2 t_{M}}{M-1}\right) & \exp \left(-j \cdot 2 \pi F_{1} \cdot \frac{2 t_{M}}{M-1}\right) & \cdots & \exp \left(-j \cdot 2 \pi F_{N} \cdot \frac{2 t_{M}}{M-1}\right) \\
\vdots & \cdots & \vdots \\
\exp \left(j \cdot 2 \pi F_{1} \cdot t_{M}\right) & \exp \left(-j \cdot 2 \pi F_{1} \cdot t_{M}\right) & \cdots & \exp \left(-j \cdot 2 \pi F_{N} \cdot t_{M}\right)
\end{array}\right]_{M \times 2 N} \\
& \mathbf{x}=\left[\frac{A_{1} P_{1}}{2} \cdot \exp \left(-j \cdot \theta_{1,0}\right) \frac{A_{1} P_{1}}{2} \cdot \exp \left(j \cdot \theta_{1,0}\right) \cdots \frac{A_{N} P_{N}}{2} \cdot \exp \left(j \cdot \theta_{N, 0}\right)\right]_{1 \times 2 N}^{H}, \\
& \mathbf{n}=\left[\begin{array}{llll}
n(1) & n(2) & \cdots & n(M)
\end{array}\right]_{1 \times M}^{T},
\end{aligned}
$$


where $\mathbf{r}$ is the satellite signal after channel serialization, and $F_{N}=f_{I F}+f_{N}$ is the coefficient in matrix $\mathbf{A}$; the unknown parameters $A_{N}$ and $\theta_{N, 0}$, contained within the column vector $\mathbf{x}$, are the satellite carrier signal intensity and initial phase, respectively. Equation (3) is the blind source separation model of the satellite signal constructed in this study by simulating multiple channels through frequency multiplication sampling. The acquisition process is the separation of matrix $\mathbf{A}$ and vector $\mathbf{x}$ from $\mathbf{r}$.

2.2. Blind Source Separation of Satellite Signals. To perform blind source separation on equation (3), the satellite carrier signal needs to be first denoised. Since threshold denoising is conceptually simple and computationally inexpensive, this study uses wavelet threshold denoising to perform noise reduction on the satellite signal. The main implementation steps of this process are outlined in the following sentences. First, wavelet transform with layer number 5 is performed on noisy signal $r$ to obtain the wavelet coefficients corresponding to the fine details $b_{k}$, and soft thresholding is then applied to $b_{k}$ to obtain the estimated wavelet coefficients $w_{k}$. Finally, wavelet reconstruction is performed using $b_{k} w_{k}$ to obtain the signal after noise reduction as the reconstructed signal $[10,11]$.

Using the reconstructed signal as the input signal $\mathbf{r}$ for the blind source separation model, simultaneously finding the autocorrelation matrix for both sides, and combining the frequency invariance of the sinusoidal signal's correlation function, the autocorrelation matrix $Q=E\left(r-r^{H}\right)$ is obtained. Decomposing the matrix $Q$ into two parts, namely, the signal subspace and the noise subspace, and performing the eigenvalue decomposition,

$$
\mathbf{Q}=\mathbf{U} \sum \mathbf{U}^{H}=\mathbf{U}_{X} \sum \mathbf{U}_{X}^{H}+\mathbf{U}_{N} \sum \mathbf{U}_{N}^{H},
$$

where the matrix $\mathbf{Q}$ has $2 N$ large eigenvalues and $M-2 N$ small eigenvalues after mathematical decomposition. $\mathbf{U}_{X}$ is the signal subspace spanned by the eigenvectors corresponding to the large eigenvalues; $\mathbf{U}_{N}$ is the noise subspace spanned by the eigenvectors corresponding to the small eigenvalues. The signal subspace and the noise subspace are mutually orthogonal; using the orthogonality of the two subspaces, the spatial spectral function can be constructed as

$$
P=\frac{1}{\mathbf{A}^{H} \mathbf{U}_{N} \mathbf{U}_{N}^{H} \mathbf{A}} \text {. }
$$

By optimizing the above spatial spectral function, the sought Doppler frequency shift of the satellite signal is obtained as the frequency corresponding to the extremum value. Finally, the matrix least squares method is used to obtain a blind estimate of the initial phase of the satellite carrier signal: $\mathbf{X}=\mathbf{A}^{\dagger} \mathbf{r}$, where $\mathbf{A}^{\dagger}$ is the pseudoinverse matrix of matrix A. Thus, the initial phase of the carrier can be solved as follows.

$$
\theta=\arctan \left[\frac{\operatorname{Im}(\mathbf{x})}{\operatorname{Re}(\mathbf{x})}\right],
$$

where $\operatorname{Im}(\mathbf{x})$ and $\operatorname{Re}(\mathbf{x})$ represent the imaginary and real parts of the complex numbers $\mathbf{x}$, respectively. The acquisition of the satellite signal is completed once both the Doppler frequency and phase of the satellite carrier signal have been obtained.

\section{Results and Discussion}

The dataset used consists of actual GPS signals collected by the University of Colorado Boulder and is named "GPSdataDiscrete Components-fs38_192-if9_55.bin.” The GPS signal is quantized with 8 bit, IF signal frequency of $9.548 \mathrm{MHz}$, sampling frequency of $38.192 \mathrm{MHz}$, carrier frequency of $1.023 \mathrm{MHz}, \mathrm{C} / \mathrm{A}$ code length 1023 , and downsampling factor of 11 . The signal consists of 8 channels, from which 32 satellites may be acquired. The signal acquisition threshold is set to 2.5. Figure 1 shows the results of satellite acquisition based on the blind source separation method. The PRN number represents the GPS satellite serial number. From figure, we can see that the method proposed in this study resulted in the successful acquisition of the signal from 8 visible satellites, for which the acquisition peaks are all greater than the preset threshold of 2.5. The remaining satellite signals with correlation peaks less than 2.5 were not acquired. The acquired satellite Doppler frequency and code phase parameters are accurate, with both the frequency error and code phase error being controlled within the allowable limits to meet the requirements of subsequent tracking.

In the following paragraphs, satellite PRN21 is used as an example to analyze the acquisition accuracy of the method proposed in this study and the parallel code phase search based on fast Fourier transform (FFT). Figure 2(a) shows the acquisition results obtained using the parallel search algorithm, and Figure 2(b) shows the results obtained using the acquisition algorithm proposed in this study. The Doppler frequency shift can be evaluated by subtracting the IF signal frequency result from $9.548 \mathrm{MHz}$, which can be used for quantifying the algorithm performance. From figures, it can be seen that the IF signal frequency obtained by the parallel code phase search algorithm is $9.54743 \mathrm{MHz}$, which has a Doppler frequency shift of $-570 \mathrm{~Hz}$ in comparison with the actual IF signal frequency. The IF signal frequency estimated by the acquisition algorithm in this study is $9.54825 \mathrm{MHz}$, which has a Doppler frequency shift of $-250 \mathrm{~Hz}$. Hence, it can be seen that the algorithm proposed in this study has a more accurate estimation of the Doppler frequency shift.

The execution times of the algorithms proposed in this study and the parallel search algorithm were statistically tested under the same environment for a total of 10 measurements. The average values were calculated, and the results obtained are given in Table 1. As given in Table 1, the execution time of the acquisition algorithm proposed in this study is much smaller than the execution time of the traditional parallel acquisition algorithm; thus, the acquisition speed of the algorithm proposed in this study is fast.

To verify the sensitivity of the acquisition algorithm, signals with different signal-to-noise ratios need to be acquired for testing. Since the flexible control of the signal-tonoise ratio cannot be realized on physically acquired data, the software Matlab was used to generate simulated GPS signals as the test signals. The simulation generates the No. 6 


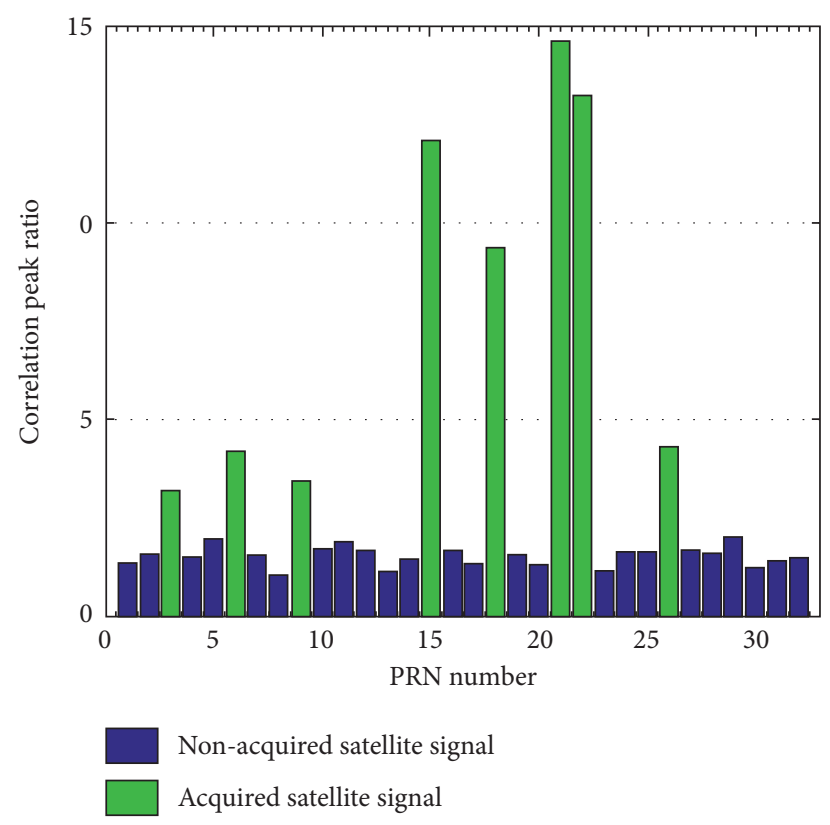

FIGURE 1: Signal acquisition results on actual collected GPS signals.

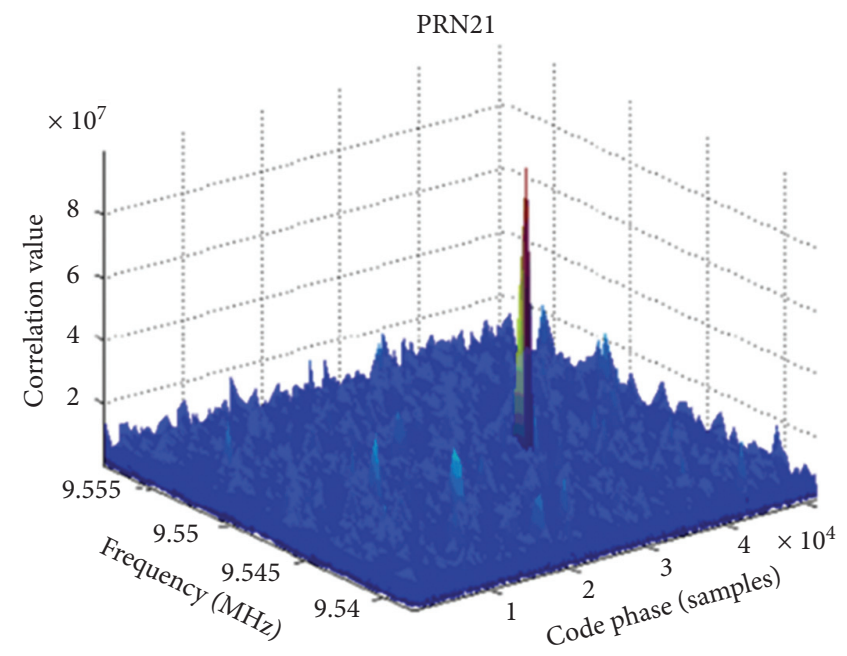

(a)

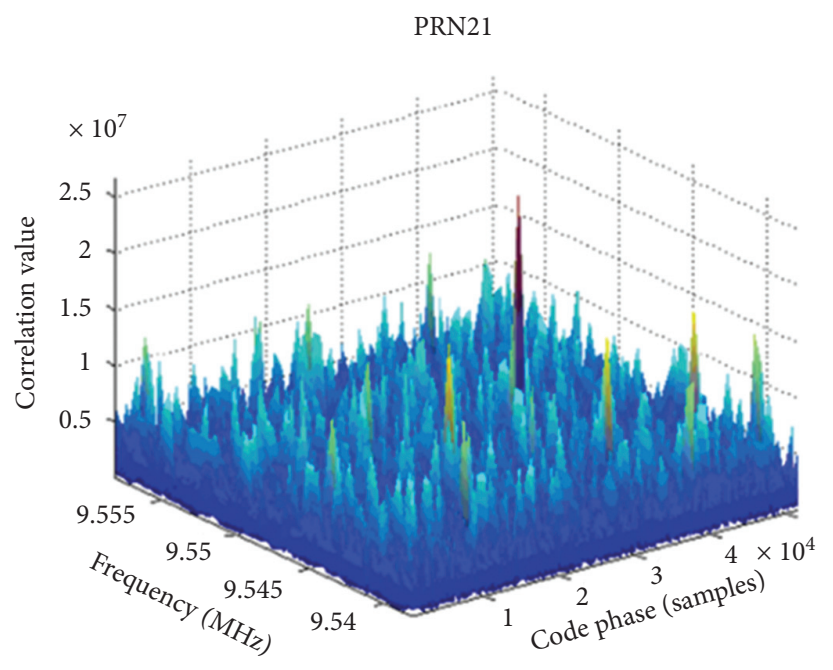

(b)

Figure 2: Acquisition results (a) for the parallel search and (b) for the proposed method.

TABLE 1: Comparison of algorithm execution times.

\begin{tabular}{lcc}
\hline Frequency search step $(\mathrm{Hz})$ & $\begin{array}{c}\text { Parallel } \\
\text { search }(\mathrm{s})\end{array}$ & $\begin{array}{c}\text { Proposed method } \\
(\mathrm{s})\end{array}$ \\
\hline 1000 & 10.415 & 0.256 \\
500 & 19.783 & 0.598 \\
\hline
\end{tabular}

GPS satellite signal with a data length of $100 \mathrm{~ms}$, randomized navigation message data, signal frequency of $4 \mathrm{MHz}$, sampling frequency of $16 \mathrm{MHz}$, and initial signal phase of 0 . The signal is combined with additive zero-mean Gaussian white noise with noise variance $\sigma_{2}=1$. Figure 3 shows the performance comparison of using the parallel code phase search acquisition algorithm using the acquisition algorithm proposed in this study to acquire the simulated GPS satellite signal.

As shown in figure, the probability of a correct acquisition increases with the increase of signal-to-noise ratio for both algorithms; however, compared with the traditional parallel acquisition algorithm, the acquisition performance of the proposed algorithm is better and more sensitive for any given signal-to-noise ratio. For the probability of correct acquisitions to reach $90 \%$, the traditional parallel acquisition algorithm requires a signal-to-noise ratio of $-41 \mathrm{~dB}$ or more, while the acquisition algorithm used in this study requires a signal-to-noise ratio of only $-44 \mathrm{~dB}$. This demonstrates that 


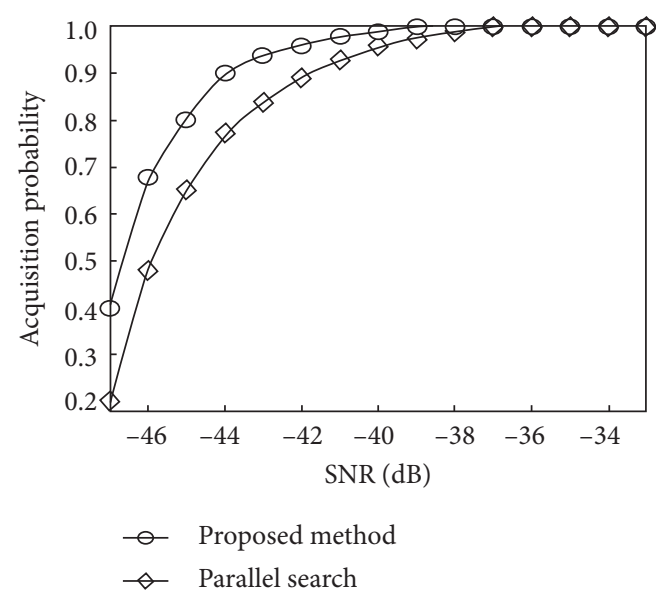

FIgURE 3: Performance comparison of acquisition algorithms.

the acquisition algorithm used in this study is more suitable for the acquisition of weak signals as compared to the traditional algorithm.

\section{Conclusion}

In order to meet the requirement of precise navigation in a real time, the compromise between acquisition speed and acquisition accuracy needs to be addressed. In this work, we proposed a new method to capture the satellite with both high accuracy and fast satellite signal acquisition. Unlike the traditional algorithm, e.g., parallel searching based on Fourier transform, the proposed method employs an idea of signal optimization where the Doppler frequency can be analytically calculated. Based on the mixture signal from multiple satellites and the time-dependent amplitude, we implemented the frequency multiplication sampling to generate the standard sinusoidal signal and then established the signal acquisition model according to blind source separation. After that, the signal background noise is reduced by introducing the wavelet denoised method where a wavelet threshold is preset. This can enhance the signal-tonoise ratio, thereby improving the acquisition performance of the satellite receiver. Finally, the subspace-based estimation algorithm was used to blindly estimate the Doppler frequency and phase of the satellite signal so as to overcome the technical limitations faced by existing acquisition methods, using which a simultaneously fast and accurate acquisition of satellite signals cannot be achieved. The experimental results from GPS satellite signal acquisition demonstrate that, as compared with the traditional acquisition methods, the new acquisition algorithm proposed in this study can achieve high accuracy and fast acquisition of the satellite signal. Furthermore, it can effectively acquire weak signals, making it more suited for high-sensitivity software receivers.

\section{Data Availability}

The datasets used and/or analyzed during the current study are available from the corresponding author upon request.

\section{Conflicts of Interest}

The authors declare that they have no conflicts of interest.

\section{Acknowledgments}

The authors acknowledge the National Natural Science Foundation of China (61973087) and the Key Research and Development Program of Guangdong Province (2019B010141001).

\section{References}

[1] D. Kaplan and J. Hegarty, Understanding GPS: Principles and Applications, Artech House, London, UK, 1996.

[2] Y. Rubinpur and S. Toledo, Signal Processing for a Reverse-GPS Wildlife Tracking System: CPU and GPU Implementation Experiences, Concurr. Comp-Pract. E., NJ, USA, 2021.

[3] K. Vasudevan, "Coherent detection of turbo-coded OFDM signals transmitted through frequency selective Rayleigh fading channels with receiver diversity and increased throughput," Wireless Personal Communications, vol. 82, no. 3, pp. 1623-1642, 2015.

[4] M. A. Farhad, M. R. Mosavi, A. A. Abedi, and K. Mohammadi, "Increasing the resistance of GPS receivers by using a fuzzy smart estimator in weak signal conditions," Journal of Navigation, vol. 73, no. 5, pp. 991-1013, 2020.

[5] S. Jing and W. Zhang, "Fast and precise acquisition of GPS signal optimized by improved orthogonal search," Laser and Optoelectronics Progress, vol. 57, no. 13, Article ID 131206, 2020.

[6] S. F. Ahamed, G. S. Ganesh, and L. Gabeshc, "Fast acquisition of GPS signal using FFT decomposition," Procedia Computer Science, vol. 87, pp. 190-197, 2016.

[7] H. Cui, Z. Li, and Z. Dou, "Fast acquisition method of GPS signal based on FFT cyclic correlation," International Journal of Communications, Network and System Sciences, vol. 10, no. 8, pp. 246-254, 2017.

[8] Y. Xie, K. Xie, and S. Xie, "Underdetermined blind separation of source using lp-norm diversity measures," Neurocomputing, vol. 411, pp. 259-267, 2020.

[9] J. Henríquez and W. Kristjanpoller, "A combined Independent Component Analysis-Neural Network model for forecasting exchange rate variation," Applied Soft Computing, vol. 83, Article ID 105654, 2019.

[10] J. Li, Y. Li, Y. Li, and Z. Qian, "Downhole microseismic signal denoising via empirical wavelet transform and adaptive thresholding," Journal of Geophysics and Engineering, vol. 15, no. 6, pp. 2469-2480, 2018.

[11] Q. Peng, T. Yu, W. Luo et al., "An adaptive iteratively weighted half thresholding algorithm for image compressive sensing reconstruction, communications signal processing and systems," in Proceedings of the 2018 CSPS, Dalin, China, July 2018 . 